\title{
Thirty-two phase sequences design with good autocorrelation properties
}

\author{
S P SINGH ${ }^{1}$ and $\mathrm{K}$ SUBBA RAO ${ }^{2}$ \\ ${ }^{1}$ Mahatma Gandhi Institute of Technology (MGIT), Gandipet, Hyderabad 500075 \\ ${ }^{2}$ Electronics and Communication Engineering Department, College of \\ Engineering, (Osmania University), Hyderabad 500012 \\ e-mail: singh_spgahlot@ rediffmail.com
}

MS received 8 January 2008; revised 22 September 2009; accepted 27 September 2009

\begin{abstract}
Polyphase Barker Sequences are finite length, uniform complex sequences; the magnitude of their aperiodic autocorrelation sidelobes are bounded by 1 . Such sequences have been used in numerous real-world applications such as channel estimation, radar and spread spectrum communication. In this paper, thirty-two phase Barker sequences up to length 24 with an alphabet size of only 32 are presented. The sequences from length 25 to 289 have autocorrelation properties better than well-known Frank codes. Because of the complex structure the sequences are very difficult to detect and analyse by an enemy's electronic support measures (ESMs). The synthesized sequences are promising for practical application to radar and spread spectrum communication systems. These sequences are found using the Modified Simulated Annealing Algorithm (MSAA). The convergence rate of the algorithm is good.
\end{abstract}

Keywords. Barker codes; Hamming scan; polyphase code; radar signal design; simulated annealing.

\section{Introduction}

Sequences with low aperiodic autocorrelation sidelobe levels are useful for channel estimation, radar, and spread spectrum communication applications. Sequences achieving the minimum peak aperiodic autocorrelation sidelobe level one are called Barker Sequences. Let $S$ be the sequence of length ' $N$ '.

$$
S=[s(0), s(1), s(1), \ldots, s(N-1)] .
$$

The aperiodic autocorrelation function (ACF) of the sequence $S$ is given by,

$$
A(k)= \begin{cases}\sum_{n=0}^{N-k-1} s(n) s^{*}(n+k) ; & 0 \leq k \leq N-1 \\ \sum_{n=0}^{N+k-1} s(n) s^{*}(n-k) ; & -N+1 \leq k \leq 0 .\end{cases}
$$


If all the sidelobes of the ACF of any polyphase sequence are bounded by

$$
|A(k)| \leq 1, \quad 1 \leq|k| \leq N-1,
$$

then the sequence is called a generalized Barker sequence or a polyphase Barker sequence.

Barker (1953) introduced binary sequences for lengths $N=2,3,4,5,7,11$, and 13, fulfilling the condition in (3). The binary Barker sequences can be regarded as a special case of polyphase Barker sequences. If the sequence elements are taken from an alphabet of size $M$, consisting of the $M^{\text {th }}$ roots of unity,

$$
s_{m}=\exp \left\{2 \pi i \cdot \frac{m}{M}\right\}=: \exp \left(i \phi_{m}\right) \quad 0 \leq m \leq M-1,
$$

the sequence is alternatively named an $M$-phase Barker sequence.

Golomb \& Scholtz (1965) investigated generalized Barker sequences and presented six phase Barker sequence of lengths $N \leq 13$. Zhang \& Golomb (1989) investigated sixtyphase Barker sequences up to length 18. For large alphabet sizes, the list of known polyphase Barker sequences was extended up to length 25 by Bomer \& Antweiler (1989). Polyphase Barker sequences of lengths up to 31 were given by Friese \& Zottmann (1994); Zhang \& Golomb (1996), wherein, at length 30, an alphabet size of 7200 had to be used. Friese (1996) presented polyphase Barker sequences up to lengths 36 wherein, at length 35 an alphabet size of 11520 were necessary. Bomer \& Antweiler (1989) presented polyphase Barker sequences of lengths 37-45. Peter Browein \& Ron Fergusion (2005) presented polyphase Barker sequences of lengths 46-63, wherein, a maximum alphabet size of 2000 had to be used. Recently Correll J Nunn \& Coxson (2009), presented polyphase Barker sequences of lengths 64-70, $72,76 \& 77$. However, polyphase Barker sequences for larger lengths require larger alphabets and the possibility for exhaustive search diminishes. Unfortunately, Barker sequences are very rare.

Binary code is one of the most commonly used radar pulse compression signals due to the easy signal generation and processing (Cook \& Bernfield 1967, Farnett \& Stevens 1990). Polyphase signal has larger main lobe-to-peak sidelobe ratio over binary signal of the same code length. However, design of higher length sequences is time consuming even when an optimization algorithm is used. Hence a group of sequences of different lengths can be designed a priori, stored in the radar system and used as and when required. Moreover, the operator can change the coded sequences frequently in order to avoid the enemy to decode the sequence; there by improving the ECCM facility. In addition, polyphase waveforms have a more complicated signal structure and thus, are more difficult to detect and analyse by an enemy's electronic support measures (ESMs). With the maturity of digital signal processors, the generation and processing of polyphase signals have now become easy and less costly. Further, with the use of floating point processors, the problems of finite precision, overflow, etc. can be avoided/minimized. Therefore, polyphase codes are increasingly becoming a favourable alternative to the traditional binary code for radar signal design.

\section{Thirty-two phase sequences}

The thirty-two Phase sequence of length $N$ is represented by a complex number sequence

$$
\left\{s(n)=\mathrm{e}^{j \phi_{m}(n)}, \quad n=1,2, \ldots, N\right\},
$$


where $\phi_{m}(n)$ is the phase of $n^{\text {th }}$ sample in the sequence and lies between 0 and $2 \pi$. If the number of the distinct phases available to be chosen for each sample in a code sequence is $M$, the phase values can only be selected from the following admissible values:

$$
\begin{aligned}
\phi_{m}(n) & \in\left\{0, \frac{2 \pi}{M}, 2 \frac{2 \pi}{M}, \ldots,(M-1) \frac{2 \pi}{M}\right\} \\
& =\left\{\psi_{1}, \psi_{2}, \ldots, \psi_{M}\right\} .
\end{aligned}
$$

For example, if $M=4$, then values of $\left\{\psi_{1}, \psi_{2}, \psi_{3}\right.$ and $\left.\psi_{4}\right\}$ will be $0, \pi / 2, \pi$ and $3 \pi / 2$ respectively.

\section{Discriminating factor (DF)}

Discrimination factor is the measure of goodness of design of any sequence. The discriminating factor (DF) as defined by Golay is as follows,

$$
D F=\frac{A(0)}{\max _{k \neq 0}|A(k)|} \text {. }
$$

The denominator is the peak sidelobe value and is related to the $L_{\infty}$ norm of the sidelobes. However, Brenner (1998) has also found polyphase sequences with low $L_{2}$ and $L_{1}$ norms. For Barker sequences, DF is equal to the length of the sequence. Higher the DF, lower is the energy required to detect a target. In addition, lower Radar Cross Section (RCS) target can be detected easily. Hence DF should be as high as possible for a given code in all conditions. Maximization of DF can be viewed as a multivariable optimization problem. In this problem, cost function is taken as the sum of squares of autocorrelation sidelobe peaks. Hence, from (2) the cost function can be written as,

$$
E=\sum_{k=1}^{N-1}|A(k)|^{2} .
$$

The minimization of cost function in (8) generates the best thirty-two phase sequence that maximized the DF.

\section{Simulated annealing (SA) algorithm}

The SA technique, introduced by Kirkpatrick et al 1983 proved efficient and powerful tool to find optimal or near optimal solutions for complex multivariable nonlinear functions. The major advantage of the SA algorithm is the ability to avoid becoming trapped in local optima during the search process. The algorithm employs a random variable search that not only accepts the changes that decrease the cost function, but also accepts some changes that increase it with a probability

$$
\rho= \begin{cases}\exp \left(-\Delta E / T_{i}\right), & \Delta E>0 \\ 1, & \Delta E<0,\end{cases}
$$

where $\Delta E$ is the change in cost due to mutation. $T_{i}$ is the control parameter, which by analogy is known as the system 'temperature.' Normally, the temperature $T_{i}$ is slowly decreased from a large value to a very small one during the annealing process. The SA algorithm can find the global optimum of a nonlinear multivariable function by carefully controlling the rate of decrease of the system temperature $T_{i}$. 


\section{Hamming scan algorithm}

The Hamming scan algorithm is a traditional greedy optimization algorithm, which searches in the neighbourhood of the point in all directions to minimize the cost function and has fast convergence rate. The basic difference between genetic algorithm and Hamming scan algorithm is that genetic algorithm uses random but possibly multiple mutations. Mutation is a term metaphorically used for a change in an element in the sequence. For example if the phase value of a thirty-two-phase sequence is $\phi_{m}(0 \leq m \leq 31)$, it is replaced with phase $\phi_{i}, i=0,1,2, \ldots, 31 i \neq m$, and the cost function for each $\phi_{i}$ change is evaluated. If the cost is reduced due to a change in phase value, the new phase value is accepted; otherwise, the original phase value is retained. This process is recursively applied to all the elements of $S$ until there is no change in the cost function. The Hamming scan algorithm looks at all the first order-Hamming neighbours of the given sequence. Thus, Hamming scan performs recursively local search among all the Hamming-1 neighbours of the sequence and selects the one whose objective function value is minimum.

\section{Thirty-two phase sequences design using MSAA}

The flowchart of MSAA for optimizing the thirty-two phase codes is shown in figure 1. The MSAA is a combination of both SA and Hamming scan algorithms. It combines the good methodologies of the two algorithms like global minimum converging property of SA algorithm and fast convergence rate of Hamming scan algorithm. The demerit of Hamming scan algorithm is that it gets stuck in the local minimum point because it has no way to distinguish between local minimum point and a global minimum point. Hence, it is suboptimal (Moharir et al 1996; 1997). The drawback in SA is that it has a slow convergence rate because even though it may get closer to the global minimum point, it may skip it because of the methodology it employs, selecting the sequences randomly and accepting them with probability based on annealing schedule. The MSAA overcomes these drawbacks (Singh \& Subba Rao 2005; 2006). The computational cost for searching the best thirty-two phase sequence of length $N$, through an exhaustive search, i.e. minimizing (8), is of the order of $(32)^{\mathrm{N}}$ and grows exponentially with the code length. Therefore, optimization of polyphase phase sequences is an NP-complete problem. The phase values of a thirty-two phase sequence set $S$ is 'mutated' as follows. First, a polyphase sequence set $S$ is randomly chosen; then the selected phase value is replaced with a phase value randomly chosen from the other $M-1$ possible distinct phase values. Now the cost function for the new code set is evaluated according to (8). If the new cost value is reduced, then accept the new set. Otherwise, accept it with a probability given in (9). The probability density function for all random selections is a uniform function among all possible values.

The next step of the algorithm is to invoke the Hamming scan to find the optimum sequence in the vicinity of sequence obtained by SA. To successfully implement the MSAA, one needs to determine few key parameters or criteria for the annealing process. These are the starting temperature, the rate of decrement of temperature, i.e. cooling schedule, the determination of equilibrium condition at each temperature, and the annealing stopping criterion.

In this work, the starting temperature $T_{0}$ is decided based on the standard deviation $\sigma$ of the initial cost distribution by setting,

$$
T_{0}=15 \sigma
$$




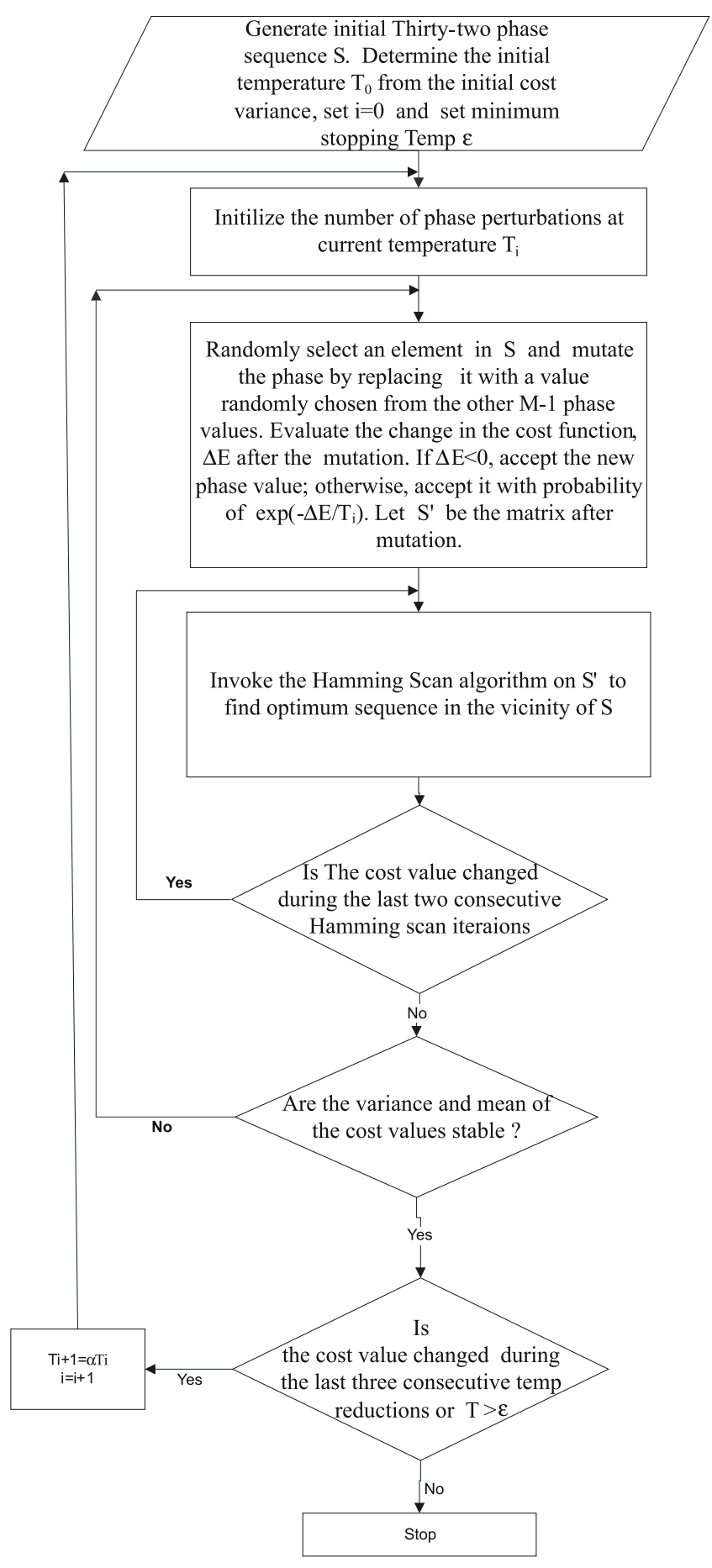

Figure 1. Flow chart of modified simulated annealing algorithm. 
From the initial temperature $T_{0}$, the system temperature is systematically reduced in each iteration according to

$$
T_{i+1}=\alpha T_{i}(0<\alpha<1),
$$

where $\alpha$ is a constant and chosen to be 0.95 in this design. At temperature $T_{i}(i>0)$, the alphabets of the sequence are constantly 'mutated' and accepted with a probability according to (9), until the cost function distribution reaches equilibrium state. Then, the temperature is reduced to $T_{i+1}$ according to (11), and the 'mutations' process is repeated until a new equilibrium state is reached at the updated temperature. The annealing process is stopped if no 'mutated' phase is accepted during three consecutive temperature reductions or $T<\varepsilon$, where $\varepsilon$ is the minimum stopping temperature. The value of $\varepsilon$ is chosen as $0 \cdot 01$.

\section{Results and discussion}

In this paper, all the autocorrelation sidelobe peaks (ASP) are single realizations obtained using Pentium - IV, processor. We have found nearly Barker codes up to length 24 (except length 22) with maximum alphabet size of only 32 , which was not reported earlier in the literature. The Barker sequences are presented in table 1. In the table, column 1 shows sequence length, $N$, column 2 shows maximum autocorrelation sidelobe peak (ASP) and column 3 shows value of ' $m$ ', used in (4). The elements of sequence are found using (4). For example, in table 1 , for sequence of length $N=4$, (there are four complex elements in the sequence) the values of $m$ are equal to $17,6,4,12$ and $M=32$. By substituting these values in the

Table 1. Correlation properties of thirty-two phase synthesized sequences.

\begin{tabular}{lll}
\hline $\begin{array}{l}\text { Sequence } \\
\text { length }(N)\end{array}$ & ASP & \\
\hline 2 & $1 \cdot 0$ & 12,12 \\
3 & $1 \cdot 0$ & $12,28,28$ \\
4 & $1 \cdot 0$ & $17,6,4,12$ \\
5 & $1 \cdot 0$ & $12,3,26,1,8$ \\
6 & $1 \cdot 0$ & $16,19,16,9,27,9$ \\
7 & $1 \cdot 0$ & $4,28,31,23,3,4,16$ \\
8 & $1 \cdot 0$ & $24,20,20,15,31,22,2,9,20$ \\
9 & $1 \cdot 0$ & $16,14,16,13,31,24,6,15,28$ \\
10 & $1 \cdot 0$ & $16,24,4,22,1,30,8,3,27,24$ \\
11 & $1 \cdot 0$ & $28,23,17,11,19,16,26,26,6,20,0$ \\
12 & $1 \cdot 0$ & $16,24,28,31,4,24,31,19,25,16,8,28$ \\
13 & $1 \cdot 0$ & $28,24,23,23,14,29,6,26,10,9,22,23,4$ \\
14 & $1 \cdot 0$ & $25,1031,6,27,14,9,4,10,4,5,16,25,30$ \\
15 & $1 \cdot 0$ & $11,30,5,26,22,20,8,22,19,5,16,17,19,21,29$ \\
16 & $1 \cdot 0$ & $7,23,9,31,15,25,1,17,23,27,26,3,4,29,24,20$ \\
17 & $1 \cdot 0$ & $16,26,2,3,15,24,12,23,1,28,27,28,21,3,26,17,8$ \\
18 & $1 \cdot 0$ & $20,4,17,5,16,1,1,15,27,27,13,8,29,25,26,30,2,1$ \\
19 & $1 \cdot 0$ & $4,22,16,3,31,17,28,10,7,19,28,21,29,7,9,4,6,0,4$ \\
20 & $1 \cdot 0$ & $30,7,15,23,29,21,25,14,10,10,9,23,11,18,10,23,20,11,30,18$ \\
21 & $1 \cdot 0$ & $15,12,5,6,9,30,27,11,6,29,3,8,13,8,20,19,0,14,30,14,25$ \\
23 & $1 \cdot 0$ & $21,0,16,28,2,11,27,16,27,21,0,5,7,5,29,4,0,20,17,5,29,21,21$ \\
24 & $1 \cdot 0$ & $29,13,28,14,26,5,16,22,30,15,12,0,24,14,8,9,5,30,2,2,10,10,16,15$ \\
& &
\end{tabular}

*The value of $m$ is used in (4) to find the sequence element $s_{m}$ 
Table 2. Autocorrelation sidelobe peaks (ASPs) and Discriminating Factors (DFs) of thirty-two-phase synthesized sequences with sequence length, $N$ varying from 2 to 1000.

\begin{tabular}{|c|c|c|c|c|c|c|c|c|}
\hline $\begin{array}{l}\text { Sequence } \\
\text { length } \\
\text { (1) }\end{array}$ & $\begin{array}{l}\text { ASP } \\
(2)\end{array}$ & $\begin{array}{l}\text { DF } \\
\text { (3) }\end{array}$ & $\begin{array}{l}\text { Sequence } \\
\text { length } \\
\text { (1) }\end{array}$ & $\begin{array}{c}\text { ASP } \\
(2)\end{array}$ & $\begin{array}{l}\text { DF } \\
\text { (3) }\end{array}$ & $\begin{array}{l}\text { Sequence } \\
\text { length } \\
\text { (1) }\end{array}$ & $\begin{array}{c}\text { ASP } \\
(2)\end{array}$ & $\begin{array}{l}\text { DF } \\
\text { (3) }\end{array}$ \\
\hline 2 & 1.0 & $2 \cdot 0$ & 40 & $1 \cdot 81$ & 22.05 & 81 & 2.44 & 33.09 \\
\hline 3 & 1.0 & $3 \cdot 0$ & 41 & 1.85 & 22.06 & 83 & 2.73 & $30 \cdot 34$ \\
\hline 4 & 1.0 & 4.0 & 42 & 2.05 & $20 \cdot 41$ & 84 & 2.53 & 33.08 \\
\hline 5 & 1.0 & 5.00 & 43 & 2.09 & 20.50 & 85 & 2.34 & $36 \cdot 19$ \\
\hline 6 & 1.0 & 5.63 & 44 & 1.80 & 24.38 & 86 & 2.84 & $30 \cdot 22$ \\
\hline 7 & 1.0 & 7.00 & 45 & 2.0 & 22.47 & 87 & $2 \cdot 30$ & 37.75 \\
\hline 8 & 1.0 & 8.00 & 46 & 1.77 & 25.85 & 88 & 2.70 & 32.54 \\
\hline 9 & 1.0 & $9 \cdot 00$ & 47 & $2 \cdot 60$ & 18.06 & 91 & 2.58 & $35 \cdot 27$ \\
\hline 10 & 1.0 & $10 \cdot 00$ & 48 & 1.91 & 25.08 & 92 & 2.62 & 35.06 \\
\hline 11 & 1.0 & 11.00 & 49 & 1.90 & 25.77 & 93 & $2 \cdot 86$ & 32.46 \\
\hline 12 & 1.0 & 12.00 & 50 & 2.08 & 23.95 & 94 & 3.08 & 30.43 \\
\hline 13 & 1.0 & $13 \cdot 00$ & 51 & $2 \cdot 0$ & $25 \cdot 61$ & 95 & $3 \cdot 17$ & 29.92 \\
\hline 14 & 1.0 & 14.00 & 52 & 1.99 & $26 \cdot 12$ & 96 & 2.69 & 35.68 \\
\hline 15 & 1.0 & 14.99 & 53 & 1.96 & 26.96 & 97 & 2.58 & 37.46 \\
\hline 16 & 1.0 & $15 \cdot 83$ & 54 & 1.71 & 31.45 & 99 & 2.90 & 34.08 \\
\hline 17 & 1.0 & $17 \cdot 00$ & 55 & 2.08 & $26 \cdot 37$ & 100 & 2.67 & $38 \cdot 11$ \\
\hline 18 & 1.0 & $17 \cdot 36$ & 56 & $2 \cdot 26$ & 24.67 & 101 & 2.58 & 39.06 \\
\hline 19 & 1.0 & 19.00 & 57 & $2 \cdot 06$ & 27.56 & 102 & 2.61 & 39.08 \\
\hline 20 & 1.0 & $19 \cdot 51$ & 58 & 1.99 & 29.09 & 121 & 3.35 & 36.09 \\
\hline 21 & 1.0 & $20 \cdot 56$ & 59 & $2 \cdot 06$ & 28.52 & 144 & 3.51 & $40 \cdot 94$ \\
\hline 22 & $1 \cdot 1$ & $19 \cdot 81$ & 60 & $2 \cdot 20$ & $27 \cdot 16$ & 169 & 3.78 & 44.67 \\
\hline 23 & 1.0 & $22 \cdot 37$ & 61 & $2 \cdot 11$ & 28.81 & 196 & 4.42 & $44 \cdot 32$ \\
\hline 24 & $1 \cdot 0$ & $22 \cdot 61$ & 62 & $2 \cdot 21$ & 27.97 & 225 & 4.69 & 47.92 \\
\hline 25 & 1.15 & $21 \cdot 58$ & 63 & $2 \cdot 41$ & 26.09 & 256 & 5.03 & $50 \cdot 88$ \\
\hline 26 & $1 \cdot 17$ & 22.08 & 64 & $2 \cdot 14$ & 29.88 & 289 & 5.06 & 57.06 \\
\hline 27 & $1 \cdot 21$ & $22 \cdot 27$ & 65 & 2.62 & 24.76 & 324 & 5.96 & $54 \cdot 30$ \\
\hline 28 & $1 \cdot 34$ & $20 \cdot 76$ & 66 & $2 \cdot 31$ & 28.51 & 361 & 7.05 & 51.77 \\
\hline 29 & 1.22 & $23 \cdot 71$ & 67 & $2 \cdot 30$ & 29.03 & 400 & 7.37 & $54 \cdot 21$ \\
\hline 30 & 1.66 & 18.05 & 68 & 2.48 & $27 \cdot 34$ & 441 & 8.47 & 52.05 \\
\hline 31 & 1.70 & $18 \cdot 17$ & 69 & $2 \cdot 25$ & 30.65 & 500 & $7 \cdot 70$ & 64.92 \\
\hline 32 & 1.53 & $20 \cdot 79$ & 70 & $2 \cdot 11$ & $33 \cdot 16$ & 550 & 8.61 & 63.87 \\
\hline 33 & 1.92 & $17 \cdot 10$ & 71 & 2.35 & $30 \cdot 15$ & 600 & $9 \cdot 12$ & 65.72 \\
\hline 34 & $1 \cdot 35$ & $25 \cdot 07$ & 72 & 2.33 & 30.88 & 650 & 9.79 & $66 \cdot 39$ \\
\hline 35 & 1.53 & 22.83 & 73 & 2.07 & $35 \cdot 16$ & 700 & $10 \cdot 21$ & 66.09 \\
\hline 36 & 1.55 & $23 \cdot 15$ & 74 & $2 \cdot 29$ & 32.23 & 800 & 11.45 & $69 \cdot 86$ \\
\hline 37 & 1.44 & $25 \cdot 64$ & 75 & 2.40 & 31.18 & 900 & 12.52 & $71 \cdot 12$ \\
\hline 38 & 1.80 & 21.03 & 76 & $2 \cdot 41$ & 31.41 & 1000 & 12.40 & $80 \cdot 60$ \\
\hline 39 & 1.61 & $24 \cdot 21$ & 79 & 2.65 & $29 \cdot 38$ & & & \\
\hline
\end{tabular}

equation we can find the elements of sequence as $-0.9808-0.1951 i, 0.3827+0.9239 i$, $0.7071+0.7071 i,-0.7071+0.7071 i$. Like wise elements of all sequences of table 1 can be found. The sequences are optimized with respect to minimizing ASP or maximizing DF. We found that the optimum solution sequence need not be unique. For example, there are two best sequences at length $N=7$ with same ASP. The values of ' $m$ ' of these sequences are equal to $4,28,31,23,3,4,16$ and $29,13,28,14,26,5,16$. For some lengths there may be more than one Barker sequence but in this paper only one is presented. For lengths from 25 to 1000 , we have found the sequences which have good correlation properties; table 2 shows 


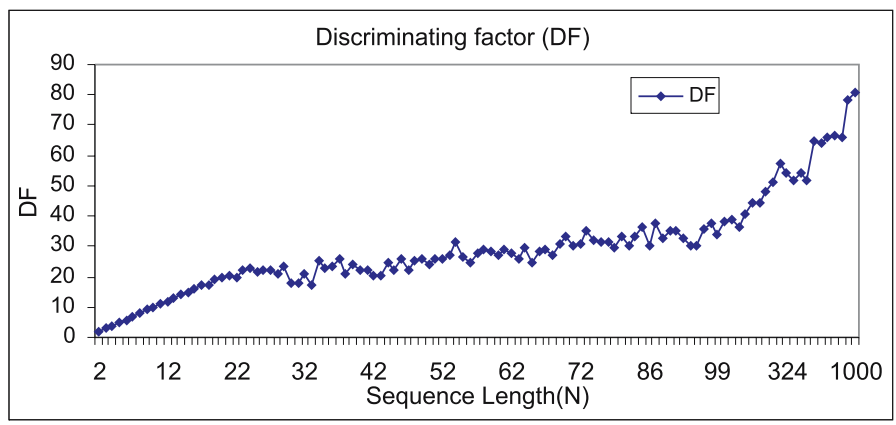

Figure 2. Discrimination factor of thirty-two phase-synthesized sequences with $N$ varying 2 to 1000 .

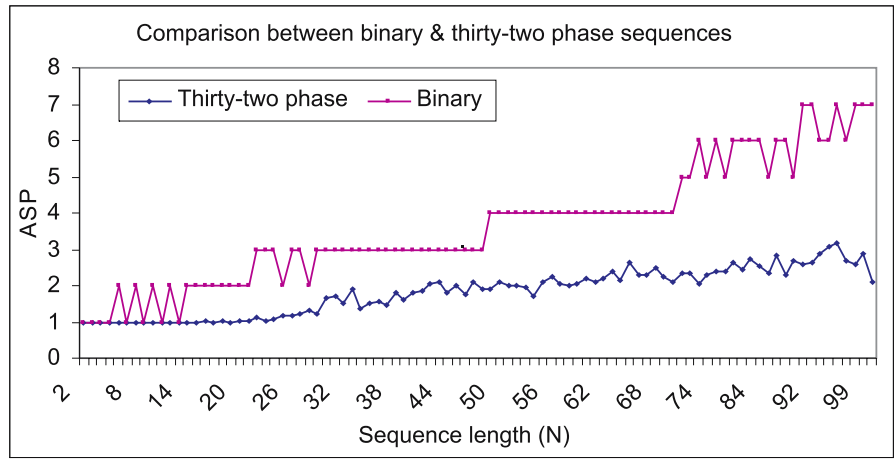

Figure 3. Comparison of autocorrelation sidelobe peaks of binary codes and thirty-two phase synthesized codes.

the synthesized results up to length 1000 . In table 2, column 1 shows sequence length, $N$, column 2 shows maximum autocorrelation sidelobe peak (ASP), and column 3 shows the Discrimination factor (DF). Figure 2 shows the discrimination factor of designed sequences.

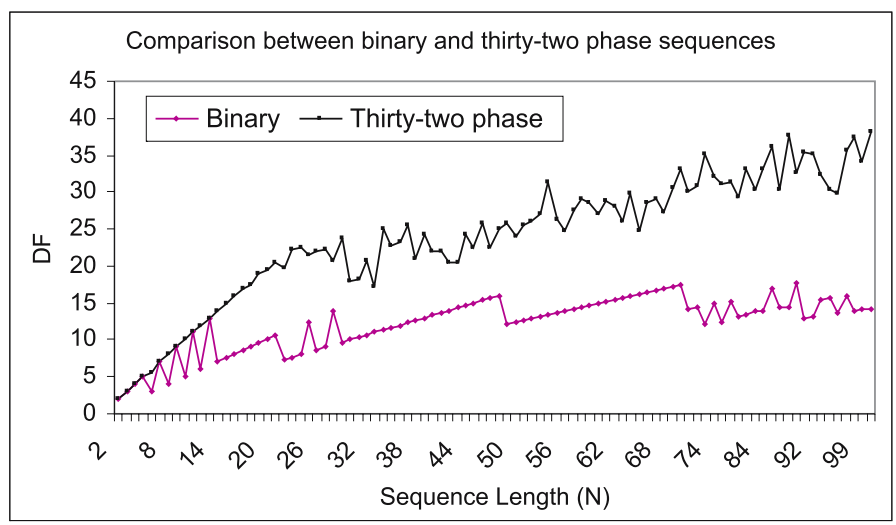

Figure 4. Comparison of discriminating factor of binary codes and thirty-two phase synthesized codes. 
Table 3. Comparison between thirty-two phase codes and Frank codes.

\begin{tabular}{|c|c|c|c|c|c|}
\hline $\begin{array}{l}\text { Sequence } \\
\text { length } \\
\text { (1) }\end{array}$ & $\begin{array}{c}\text { Frank } \\
\text { codes ASPs } \\
\text { (2) }\end{array}$ & $\begin{array}{c}\text { Synthesized } \\
\text { codes ASPs } \\
\text { (3) }\end{array}$ & $\begin{array}{l}\text { Sequence } \\
\text { length } \\
\text { (1) }\end{array}$ & $\begin{array}{c}\text { Frank } \\
\text { codes ASPs } \\
\text { (2) }\end{array}$ & $\begin{array}{l}\text { Synthesized } \\
\text { codes ASPs } \\
\text { (3) }\end{array}$ \\
\hline 16 & 1.4 & $1 \cdot 0$ & 121 & $3 \cdot 51$ & $3 \cdot 35$ \\
\hline 25 & 1.6 & 1.15 & 144 & $3 \cdot 86$ & 3.51 \\
\hline 36 & $2 \cdot 0$ & 1.55 & 169 & $4 \cdot 14$ & 3.78 \\
\hline 49 & $2 \cdot 24$ & 1.9 & 196 & 4.49 & $4 \cdot 32$ \\
\hline 64 & $2 \cdot 61$ & $2 \cdot 14$ & 225 & 4.78 & 4.69 \\
\hline 81 & 2.87 & 2.44 & 256 & $5 \cdot 12$ & 5.03 \\
\hline 100 & 3.23 & 2.62 & 289 & 5.41 & 5.06 \\
\hline
\end{tabular}

Figure 3 shows the comparison of ASPs of Binary sequences and thirty-two phase sequences and the ASPs for the later sequences is far less. Ideally, the ASP should be zero. Figure 4 shows the comparison of DFs of Binary sequences and thirty-two phase sequences. The correlation properties of the synthesized sequences are far better than Binary sequences Greg Coxson \& Jon Russo (2004), Nadav Levanon \& Eli Mozeson (2004) Matthew A Ferrara (2006). It can be seen from the figure that as the length increases, DF also increases but not linearly. Obtaining higher DF's at larger lengths is really a challenging problem. The correlation properties of these sequences are compared with well known Frank codes. Table 3 shows the maximum autocorrelation sidelobe peak (ASP) of both synthesized sequences and Frank codes for different values of $N$. In table 3 , column 1 shows sequence length, $N$, column 2 shows ASPs of Frank codes, and column 3 shows the ASPs of synthesized codes.

Figure 5 shows the comparison of ASPs of Frank codes and thirty-two phase synthesized sequences. Sequences up to length 289 have autocorrelation properties better than well-known Frank codes (1963). Apart from having better autocorrelation properties, the synthesized sequences have a more complicated signal structure than Frank codes, and thus, are more difficult to detect and analyse by an enemy's electronic support measures (ESMs). Figure 6 shows the autocorrelation function of thirty-two phase synthesized sequence of length, $N=$ 500 , which is almost like an impulse function. If the autocorrelation function of radar is impulse like then radar range resolution and parameter measuring accuracy will be good and

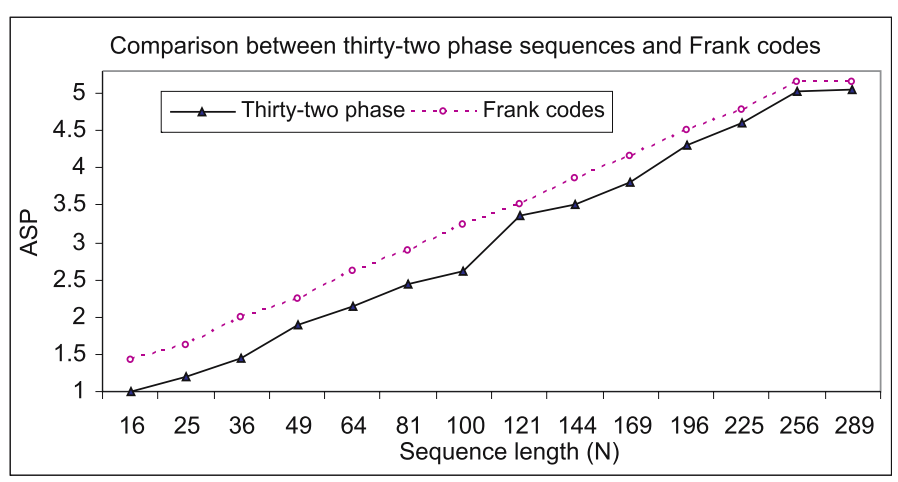

Figure 5. Comparison of autocorrelation sidelobe peaks of Frank codes and thirty-two phase synthesized codes. 


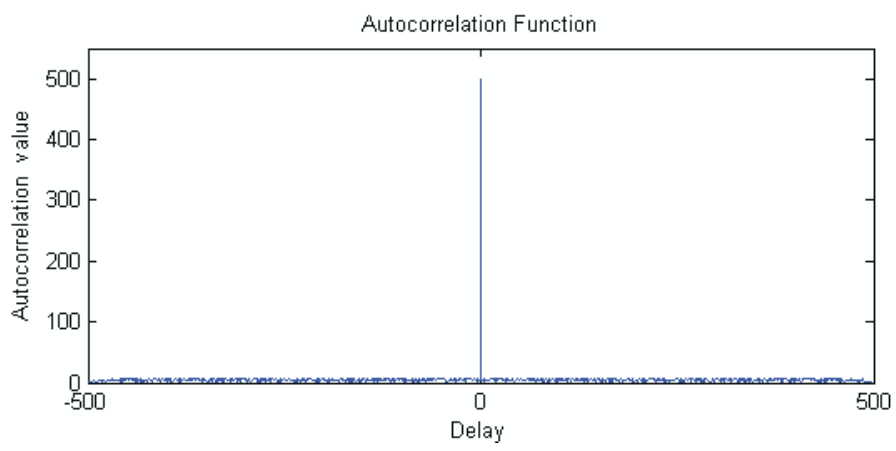

Figure 6. Autocorrelation function of thirty-two phase synthesized sequence of length $N=500$.

measurement will be unambiguous. The effect of noise is also very less on these sequences as a correlator is used in the receiver which is matched with the transmitted sequence but not with noise.

\section{Conclusions}

An effective Modified Simulated Annealing Optimization Algorithm has been used for the design of thirty-two phase-coded sequences used in radar systems and spread spectrum communications for significantly improving performance of the system. This new approach combines the SA and the Hamming scan algorithms and provides a powerful tool for the design of polyphase sequences. With the new algorithm, thirty-two phase sequences are designed for different code lengths, $N$. We have found Barker codes up to length 24 (except length 22) with a maximum alphabet size of only 32, which was not reported in the literature. Further, these sequences have correlation properties better than Frank codes. The synthesized sequences presented in this paper not only have better correlation properties but also these codes are very difficult to detect and analyse by enemy's electronic support measures (ESMs). Hence, these sequences are very useful for radar as well as spread spectrum communication systems.

\section{References}

Barker R H 1953 Group synchronizing of binary digital system in Jackson W (Ed): Communication theory (Butterworths, London) 273-287

Bomer L, Antweiler M 1989 Polyphase barker sequences. Electronics Letters 34(16): 1577-1579

Brenner A R 1998 Polyphase barker sequences upto length 45 with small alphabets. Electronics Letters 34(16): 1576-1577

Carroll J Nunn, Gregory E Coxson 2009 Polyphase pulse compression codes with optimal peak and integrated sidelobes. IEEE TAES 45(2): 775-781

Cook C E, Bernfield M 1967 An introduction to theory and application. Radar Signals (New York: Academic Press)

Farnett E C, Stevens G H 1990 Pulse compression radar. Radar Handbook, Second ed. (New York: McGraw-Hill)

Frank R L 1963 Polyphase codes with good non-periodic correlation properties. IEEE Trans. Inform. Theory IT-9: 43-45 
Friese M 1996 Polyphase Barker sequences upto length 36. IEEE Trans. Inf. Theory IT-42(4): $1248-1250$

Friese M, Zottmann H 1994 Polyphase Barker sequences upto length 31. Electronics Letters 30(23): 1930-1931

Golomb S W, Scholtz R A 1965 Generalized Barker sequences. IEEE Trans. Inf. Theory IT-11(4): $533-537$

Greg Coxson, Jon Russo 2004 Efficient exhaustive search for optimal peak sidelobe binary codes. Proceeding of IEEE : 438-443

Kirkpatrick S C D, Gelatt M P Vecchi 1983 Optimization by simulated annealing. Science 220: 671-680

Matthew A Ferrara 2006 Near optimal peak sidelobe Binary codes. Proceeding of IEEE : 400-403

Moharir P S, Singh R, Maru V M 1996 S-K-H algorithm for signal design. Electronics Letters 32(18): 1642-1649

Moharir P S, Maru V M, Singh R 1997 Bi-parental product algorithm for coded waveform design in radar. Sādhanā 22(5): 589-599

Nadav Levanon, Eli Mozeson 2004 Radar signals IEEE Press, Wiley Interscience

Peter Browein, Ron Fergusion 2005 Polyphase sequence with low autocorrelation. IEEE Trans. Inf. Theory IT-51(4): 1564-1567

Singh S P, Subba Rao K 2005 A modified simulated annealing algorithm for binary coded radar signal design. Proc. International Radar Symposium India : 693-697

Singh S P, Subba Rao K 2006 Modified simulated annealing algorithm for polyphase code design. Proc. IEEE ISIE-06 Canada : 2961-2971

Zhang N, Golomb S W 1989 Sixty phase generalized Barker sequences. IEEE Trans. Inf. Theory IT-35(4): 911-912

Zhang N, Golomb S W 1996 7200-phase generalized Barker sequences. IEEE Trans. Inf. Theory IT-35(4): 1236-1238 